\title{
6 OPEN ACCESS \\ Reducing social inequalities in health: the role of simulation modelling in chronic disease epidemiology to evaluate the impact of population health interventions
}

\author{
Brendan T Smith, ${ }^{1,2}$ Peter M Smith, ${ }^{1,2,3}$ Sam Harper, ${ }^{4}$ Douglas G Manuel, ${ }^{1,5,6,7,8}$ \\ Cameron A Mustard ${ }^{1,2}$
}

${ }^{1}$ Dalla Lana School of Public Health, University of Toronto, Toronto, Ontario, Canada ${ }^{2}$ Institute for Work \& Health, Toronto, Ontario, Canada ${ }^{3}$ School of Public Health and Preventive Medicine, Monash University, The Alfred Centre, Melbourne, Victoria, Australia ${ }^{4}$ Department of Epidemiology, Biostatistics and Occupational Health, McGill University, Montreal, Quebec, Canada ${ }^{5}$ Ottawa Hospital Research Institute, Ottawa, Ontario, Canada

${ }^{6}$ The Department of Family Medicine and the Department of Epidemiology and Community Medicine, University of Ottawa, Ontario, Canada

${ }^{7}$ C.T. Lamont Primary Health Care Research Centre and Bruyère Research Institute, Ottawa, Ontario, Canada ${ }^{8}$ The Institute for Clinical Evaluative Sciences, Toronto, Ontario, Canada

\section{Correspondence to} Brendan T Smith, Institute for Work \& Health, 481 University Ave, Toronto, ON, Canada M5G 2E9; bsmith@iwh.on.ca

Received 17 April 2013 Revised 3 October 2013 Accepted 28 November 2013 Published Online First 20 December 2013

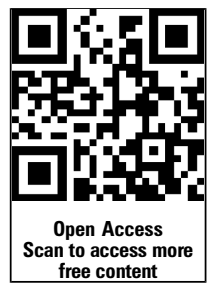

\section{ABSTRACT}

Reducing health inequalities has become a major public health priority internationally. However, how best to achieve this goal is not well understood. Population health intervention research has the potential to address some of this knowledge gap. This review argues that simulation studies can produce unique evidence to build the population health intervention research evidence base on reducing social inequalities in health. To this effect, the advantages of using simulation models over other population health intervention research methods are discussed. Key questions regarding the potential challenges of developing simulation models to investigate population health intervention research on reducing social inequalities in health and the types of population health intervention research questions that can be answered using this methodology are reviewed. We use the example of social inequalities in coronary heart disease to illustrate how simulation models can elucidate the effectiveness of a number of 'what-if' counterfactual population health interventions on reducing social inequalities in coronary heart disease. Simulation models are a flexible, cost-effective, evidencebased research method with the capacity to inform public health policy-makers regarding the implementation of population health interventions to reduce social inequalities in health.

\section{INTRODUCTION}

Reducing social inequalities in health is one of the most significant challenges facing public health today. To this effect, there has been a global call to action to reduce health inequalities. ${ }^{1}$ Most senior public health policy-makers agree that specific interventions are needed to reduce social inequalities in health. ${ }^{2}$ However, which interventions and preventive strategies will be most effective in reducing the observed inequalities are not well understood. $^{3}$ Population health intervention research (PHIR) can address some of this knowledge gap by providing evidence for decision-makers regarding the effects of policies, programmes and resource distribution strategies across all sectors that aim to improve population health ${ }^{4}$ and promote health equity. ${ }^{5}$

Simulation modelling can help build the PHIR evidence base on reducing social inequalities in health. These models can help estimate the effect of a number of 'what-if' counterfactual scenarios, where the introduction of various intervention alternatives could be tested for their capacity to improve population health and promote health equity. ${ }^{6}$ To illustrate their utility, we introduce simulation models and what they add to PHIR methods. Moreover, we discuss how to develop simulation models to investigate social inequalities in health and the types of questions that can be answered using this methodology. These topics are explored using the example of social inequalities in coronary heart disease (CHD). There is increasing interest in complex systems thinking ${ }^{7}$ and more specifically social network analysis and agent-based modelling techniques ${ }^{8}$ in social epidemiology. This manuscript builds on these discussions with an in-depth description of the relevance of simulation models to PHIR.

\section{BUILDING EVIDENCE TO REDUCE SOCIAL INEQUALITIES IN HEALTH: PHIR}

Population health interventions that address the underlying social and economic conditions essential for health have the potential to make a large impact on reducing social inequalities in health. ${ }^{5}$ To date, epidemiological research has primarily focused on conducting descriptive and analytical studies to determine the extent to which social inequalities in health exist. ${ }^{4}$ Information from these studies has been invaluable to understanding this phenomenon. For example, Harper $e t a l^{9}$ have provided a detailed review of the contribution of social determinants to the decline in cardiovascular diseases during recent decades.

However, evidence is often lacking on how to proceed to reduce health inequalities. ${ }^{3}$ There are several potential targets to consider for interventions aimed at reducing social inequalities in health, ranging from micro-level (individual) to macrolevel (societal) factors. ${ }^{10}$ Therefore, a relevant question is, how do we move forward to build evidence regarding the impact of population health interventions on reducing social inequalities in health? A first step is to better understand whether current PHIR methods are adequate for providing clear solutions for taking action.

\section{PHIR methods}

Developing appropriate research methods has been a significant challenge in PHIR. ${ }^{3}$ Intervention research methods can be classified into two broad categories. The first, intervention studies, examines the accrued benefit of interventions in specific 
(study) populations, including a number of quasi-experimental (eg, natural experiments, interrupted time-series designs, regression discontinuity approaches and comparative research between similar jurisdictions) and controlled study approaches (eg, randomised control trials). The second category of intervention research examines the potential benefit of introducing interventions into a study population, mainly modelling or simulation studies. Evidence from intervention studies is essential to informing studies that model the effects of interventions. While the merits and challenges of intervention studies have been discussed in the literature, the focus of this manuscript is to review the utility of simulation modelling to advancing PHIR. Simulation modelling is complimentary, and not a replacement, to traditional epidemiological methods that examine the etiological relationships between risk factors and disease.

\section{Simulation modelling}

Epidemiological simulation models represent causal relationship between the exposure to etiological or prognostic risk factors, treatments and health outcomes. ${ }^{11}$ These methods can be applied to simulate the impact of population health interventions within and across populations over time. The use of simulation models is well established both in health (eg, economic evaluation and infectious disease) and in other research fields (eg, climate change and economics). However, simulation models remain underutilised in chronic disease research. ${ }^{12} 13$

\section{What do simulation studies add to PHIR on chronic disease?}

Simulation models are flexible, cost-effective way to explore population health interventions. A common approach is to estimate the effectiveness of a defined intervention in a real-life setting for a targeted population that is different from the original study population. For example, would introducing a policy that increases price and reduces availability of cigarettes decrease youth smoking prevalence? Another approach examines the benefit of hypothetical interventions without specifying the nature of the intervention used to achieve the desired health benefit. For example, if physical activity rates increase by $20 \%$, to what extent would heart disease prevalence change? When using this approach, the proposed benefits of hypothetical interventions need to be realistic, rooted in best-practice evidence, with careful attention paid to the different mechanisms of achieving the desired population exposure. ${ }^{14}$

From a research perspective, intervention studies provide valuable information on the effectiveness of population health interventions and will advance knowledge on how to reduce social inequalities in health. ${ }^{3}$ However, policy-makers can experience barriers translating this information into the implementation of effective population health interventions. ${ }^{2}$ Policy-makers are tasked with answering a different question than researchers. Not only whether a population health intervention is effective, but also which intervention(s) will have the greatest impact on achieving the desired health goal in their jurisdiction. In answering this question challenges arise from the limited availability of information on the benefits and costeffectiveness of all potential interventions, making it difficult to reflect on an array of intervention options, particularly without evidence about their differential impacts on social subgroups. ${ }^{15}$

Therefore, from a decision-maker's point of view, it is easy to see how researchers may often have the right answers to the wrong questions, ${ }^{2}$ and those generating the evidence (ie, social epidemiologists) may not be aware of this problem. ${ }^{16}$ Simulation models can be an invaluable tool for bridging the evidence gap between researchers and policy-makers. The contribution of both PHIR in general and more specifically simulation models to reducing this disparity is contingent on researchers asking more pragmatic, policy-focused questions to begin with. Simulation models are a platform for integrating the best available evidence from multiple studies as well as expertise from a diversity of scientific disciplines to model the social, behavioural, environmental and genetic effects on health. ${ }^{17}$ By combining these complex factors into a single model, a better understanding can be achieved of how they interact to influence population health. For example, the complex interactions between the individual, community and macro-policy level interventions available to reduce social inequalities in health. ${ }^{10}$

\section{Advantages of simulation studies for PHIR on chronic disease}

Simulation models have a number of practical advantages over other PHIR methods. They enable the synthesis of evidence from multiple data sources, improving our understanding of the complex pathways that influence population health. The process of conceptualising a simulation model linking exposures and outcomes can be invaluable in identifying gaps in the evidence base, illustrated by the lack of evidence surrounding parameters to be included in the model. ${ }^{13}$ Other types of PHIR methods may only consider the effect of a single intervention or lack the appropriate data or control to disentangle the complex factors necessary to determine the true effect of an exposure on a given health outcome, whereas by combing data from multiple sources in simulation modelling these challenges can sometimes be overcome. The benefits of multiple types of interventions and intervention strategies (eg, targeting high-risk populations vs the whole population) can be estimated. Combined, this information can help policy-makers evaluate the impact of implementing individual or combinations of population health interventions. ${ }^{17}$ In addition, simulation studies can be a costeffective approach over other PHIR designs. Moreover, simulation models can help inform interventions when the direct observation of health outcomes is not feasible, practical or ethical to perform. Therefore, this flexible, cost-effective, evidence-based research method produces relevant evidence for policy-makers to make informed decisions regarding the implementation of population health interventions.

One limitation to this approach is that the quality of simulation models is inevitably linked to that of the data, evidence, assumptions specified in the model, and that the combination of these factors may have greater than additive effects on model estimates. ${ }^{18}$ Understanding the limitations associated with the selected parameters and being transparent about potential biases is important when communicating the results from simulation models. ${ }^{17}$ Another limitation is the number of simplifying assumptions that need to be made when attempting to model reality. The degree to which these assumptions impact the overall conclusions should be tested using sensitivity analyses. ${ }^{19}$ Finally, the development, maintenance and updating of simulation models is continually required to ensure their relevancy for answering policy-relevant research questions. While this requires a significant dedication of resources, simulation modelling remains a cost-effective approach to evaluating the effect of population health interventions.

\section{BUILDING A SIMULATION MODEL TO INVESTIGATE THE IMPACT OF POPULATION HEALTH INTERVENTIONS ON SOCIAL INEQUALITIES IN CHD}

A number of methodological challenges need to be considered to enable the greater use of simulation modelling in PHIR to 
understand how to reduce social inequalities in health. To highlight some of these challenges, key questions in developing a simulation model to examine the relationship between socioeconomic position (SEP; typically measured using education, occupation and income) and CHD are discussed. The inverse association between SEP and CHD is well established across rich countries, ${ }^{9}$ and simulation studies have been applied to study CHD. ${ }^{20}$ However, few simulation studies have been extended to examine the impact of population health interventions on reducing social inequalities in CHD. ${ }^{20}$

\section{What variables should be included in the simulation model?}

The first steps in developing a simulation model are to define the scope and choose the appropriate model for the research question. The inclusion and proper parameterisation of social measures in simulation models is essential for assessing the impact of population health interventions on social inequalities in CHD. To evaluate the effect of changing a given exposure (eg, education) or mediator (eg, obesity) on CHD (eg, incidence or mortality), they all must be included in the model. Therefore, to truly investigate social inequalities in CHD, upstream determinants of health as well as factors outside of the health field need to be included in simulation models (figure 1A). This has been accomplished in simulation models examining policy levers in tobacco control, ${ }^{21}$ obesity $^{17}$ and population-based prevention. $^{22}$

The goal is to make simulation models flexible enough to evaluate interventions that target several pathways between SEP and CHD. For example, what is the impact of intervening on different social factors (eg, education vs income), different risk factors (eg, smoking vs diet), the same risk factor using different interventions (eg, mass media campaigns vs regulation), at different points in the life course (eg, childhood vs adulthood) or on targeted versus population-level interventions (figure $1 \mathrm{~B}, \mathrm{C}$ ).

\section{What mechanisms link SEP and CHD?}

The next step is to map the relationships linking SEP and CHD. The process can be invaluable in identifying gaps in the evidence base, illustrated by the uncertainty surrounding model parameters. ${ }^{13}$ Mathematical equations are used to model how these factors interact to predict CHD. To parameterise the equations, one strategy is to use information from a number of data sources, prioritising the highest level of evidence available. Alternatively, established predictive risk algorithms have been developed to predict CHD. ${ }^{23}$ While these algorithms have traditionally been developed for clinical populations, their relevance for assessing baseline risk in population settings has been demonstrated. ${ }^{24}$

Once parameters are estimated, models need to be calibrated (refinement of model parameters to reproduce expected or observed results) and validated using an external data source. ${ }^{19}$ Sensitivity analyses are also used to test the robustness of the different model parameters.

\section{Does the simulation model predict risk consistently across SEP groups?}

An important assumption when investigating inequalities is whether simulation models accurately predict CHD within different subpopulations. Evidence from CHD risk prediction algorithms has demonstrated that including SEP measures can improve model calibration in low-SEP groups ${ }^{25}$; an effect that will otherwise underestimate inequalities between SEP groups. For example, the Framingham Risk Score ${ }^{23}$ has been demonstrated to underestimate CHD risk in low-SEP individuals; a bias reduced by incorporating $\mathrm{SEP}^{26}$ Moreover, CHD risk algorithms that include area-based deprivation measures have shown improved CHD risk prediction in low-SEP individuals. ${ }^{27}$

Further research is needed on how best to include SEP into $\mathrm{CHD}$ risk prediction. The selection of SEP indicator(s) has largely been influenced by what information is available in a particular data source, a restriction that does not apply when simulating population-level data. Given the abundant evidence that multiple measures of SEP are likely necessary to capture the full range of socioeconomic differences, considering more than a single measure in simulation models seems prudent. ${ }^{28}$

\section{Is the effectiveness of population health interventions equal across social groups?}

Simulation models need to incorporate different levels of community effectiveness for interventions by social group. The 'equity effectiveness loop' describes the hypothesised differential equity effectiveness as relating to (1) lower awareness, access or coverage; (2) screening, diagnosis or targeting; and (3) compliance of providers and adherence of consumers. ${ }^{29}$ Simulation models should be user-friendly, allowing easy manipulation of these factors across SEP groups on the estimated intervention benefits.

Currently, there is a poor understanding of intervention effectiveness across social groups. ${ }^{30}$ The assessment of equity in individual studies and systematic reviews will help contribute to reducing this knowledge gap. ${ }^{31}$ Collecting data on the benefits of interventions both in terms of population and social inequalities health is important. For example, a recent systematic review that demonstrated the effectiveness of public health interventions on both population health, but also on reducing social inequalities in smoking. ${ }^{32}$ Further, systematic reviews that aggregate the effectiveness of interventions that target the wider social determinants of health will be useful in demonstrating how intervening on these factors can contribute to reducing health inequities. ${ }^{30}$ These studies provide information regarding which interventions should be considered in simulation studies that investigate solutions for reducing social inequalities in CHD.

Additionally, from a modelling perspective, assessing the effectiveness of interventions across different social groups is invaluable. For example, a recent systematic review provided evidence on the types of interventions that may, despite their best intentions, have the unintended consequence of generated inequalities between groups. ${ }^{33}$ These studies provide an enriched understanding used to parameterise models around the uptake and effectiveness of interventions.

\section{SIMULATION MODELLING: UNDERSTANDING SOLUTIONS TO REDUCE SOCIAL INEQUALITIES IN HEALTH Modelling past trends in social inequalities in CHD}

Simulation studies have been used to better understand the substantial decline in recent decades of CHD mortality across Westernised countries. ${ }^{20}$ For example, the IMPACT CHD model seeks to evaluate the contribution of the two broad categories attributed for the reduction in CHD mortality: changes in population health CHD risk factors and improvement of medical and surgical techniques. ${ }^{34}$ The contribution of these factors is now being evaluated both in terms of population health and by deprivation level. ${ }^{34}$ The findings from this model provide useful information for making decisions about future resource allocation and which targets should be prioritised to reduce the population burden and social inequalities in CHD. 
A

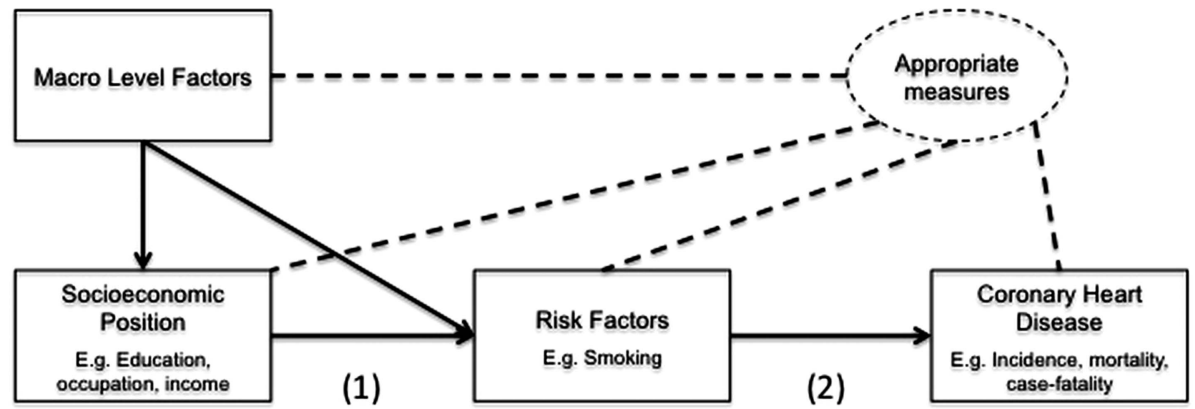

(2)

Figure 1 Building a simulation model to investigate the impact of population health interventions on social inequalities in coronary heart disease (CHD). (A) The selection of measures of inequalities, mediators and outcomes and the need to determine the causal relationships between variables, steps (1) and (2). Step (3) in (B) illustrates three different potential targets for population health interventions to reduce CHD, the effects of which can be evaluated and compared by running different 'what-if' counterfactual scenarios in the model. Step (4) in (C) represents the possibility of evaluating three different interventions for the same risk factor to evaluate which intervention will result in the greatest reduction in CHD. Interventions represented in step (4) should be parameterised to allow for different effectiveness by socioeconomic group.

\section{Evaluating the impact of interventions} on future trends in CHD

Simulation models enable the testing of multiple 'what-if' intervention scenarios with respect to future changes in CHD risk factors, the distribution of SEP in the population (eg, income distribution) and the structural determinants of health inequalities (eg, macro-level policies to improve housing). This methodology enables the testing of different strategies for achieving health equity. For example, Hoffmann et $a l^{35}$ investigated the impact of the social redistributing of $\mathrm{CHD}$ risk factors (smoking, alcohol, physical inactivity and body mass index) on mortality. This approach levels down the population prevalence of risk factors to those observed in the highest educational attainment group to understand the proportion of mortality that would be reduced if social inequalities across all risk factors were eliminated. Alvarado et $a l^{36}$ examined different scenarios for how the US Healthy People 2010 targets could be achieved and what the corresponding effect would be on population and social inequalities in CHD. In addition to levelling down, three other intervention scenarios were investigated: (1) ideal (all social groups achieve risk factors target), (2) more realistic (risk factor target is achieved, but the social gradient across risk factors remains) and (3) poverty focused (only the most disadvantaged group is targeted and benefits from a risk factor reduction). Benach et $a l^{37}$ present an example of using simulation modelling to investigate a new typology of policies to reduce social inequalities in health, defined as targeted, universal with additional targeting, redistributive and proportionate universalism.

Simulation models can also be used to estimate how introducing a set of interventions into a population can impact social inequalities in CHD. For example, in the Whitehall Study, Kivimaki et $a l^{38}$ used a risk equation approach, accounting for major CHD risk factors (systolic blood pressure, total cholesterol, blood glucose in pre-diabetic people, non-insulindependent diabetes and cigarette smoking) to determine expected CHD mortality both before and after applying bestpractice risk factor interventions. 
To date, simulation studies have focused on downstream determinants of health to understand the potential benefits of population health interventions on reducing social inequalities in CHD. These interventions may be less likely to reduce social inequalities in health and may actually increase inequalities, ${ }^{39}$ depending on whether absolute or relative inequalities are considered. ${ }^{40}$ However, these studies have demonstrated the types of questions that can be investigated using simulation models to reduce social inequalities in health. The extension of simulation models to improve modelling of more complex systems linking social exposures and disease will enhance our ability to answer both research and policy questions regarding how best to reduce social inequalities in CHD.

\section{CONCLUSION}

A gap exists between the descriptive and analytical evidence demonstrating social inequalities in health and the knowledge of which population health interventions are most likely to reduce the observed health inequalities. This mismatch of problems and solutions is not surprising, given the complexity of factors that are responsible for causing social inequalities in health. These factors can interact in non-linear ways, may only manifest over long periods of time and may change depending on the context and time period. To develop adequate population health interventions to reduce social inequalities in health, researchers will need to use appropriate methods that elucidate the causal pathways linking the social environment to health outcomes.

This manuscript argues that simulation models can provide valuable insight into which population interventions would have an optimal impact on reducing social inequalities in health. Specifically, simulation studies can estimate the historical contribution of risk factors to the observed trends in social inequalities in health. This information can be used to identify potential targets for population health interventions to reduce social inequalities in health. Moreover, simulation models can be used to forecast future trends in social inequalities in health. This feature presents a powerful tool for policy-makers and researchers alike to evaluate 'what-if' scenarios, estimating the effects of potential population health interventions on future health outcomes. These models can also be used to estimate which intervention strategies will best reduce health inequalities. The proposed methodology is not unrealistic; it is currently being applied and can be expanded to enhance our understanding of how to tackle social inequalities in health. A significant challenge in achieving health equity has been a poor understanding of the outcomes of population health interventions across populations. Simulation studies can help address this research gap by providing cost-effective, timely research methods that provide policy-makers with evidence for action to reduce social inequalities in health.

\section{What is already known on this subject?}

- Which population health interventions will be most effective in reducing social inequalities in health remains unclear.

- Simulation models can help estimate 'what-if' counterfactual scenarios, where the introduction of various intervention alternatives could be tested for their capacity to improve population health.

- The use of simulation models is well established in other fields of health research.

\section{What this study adds?}

- Simulation models can be extended to investigate the impact of population health interventions on social inequalities in health.

- This can be accomplished by incorporating multiple social exposures, accurate risk prediction within and differential effectiveness of interventions between subpopulations into simulation models.

- Several examples of counterfactual 'what-if' research questions are highlighted to demonstrate the potential for simulation models to provide evidence on how to achieve reductions in social inequalities in health.

- This manuscript provides an argument for the increased use of simulation models to provide policy-makers with evidence for action to reduce social inequalities in health.

Acknowledgements BTS is supported through a Frederick Banting and Charles Best Canada Graduate Scholarships Doctoral Award, Canadian Institutes of Health Research. In addition, BTS was a Lupina Junior Doctoral Fellow in the Comparative Program on Health and Society, Munk Centre for International Studies, University of Toronto during the conceptualization of this work. PMS is supported through a Discovery Early Career Research award from the Australian Research Council. SH was supported by a Chercheur Boursier Junior 1 from the Fonds de la Recherche en Santé du Québec. DGM holds a Chair in Applied Public Health from the Canadian Institute for Health Research and the Public Health Agency of Canada. These funding sources had no involvement in any part of the manuscript. The authors are grateful to the Simulation Technology for Applied Research (STAR) team, a CIHR new emerging team grant, for their feedback on the ideas presented in the manuscript. In particular, we would like to thank Drs Peter Tanuseputro, Deirdre Hennessy and Laurie Corna for their review and comments on the manuscript.

Contributors BTS conceptualised, drafted, reviewed and revised the final version of the manuscript as published. PMS, SH, DGM and CAM all aided in the conception and design of the manuscript, critically revised several drafts for intellectual content and approved the final version of the manuscript as published.

Funding Canadian Institutes of Health Research.

\section{Competing interests None.}

Provenance and peer review Not commissioned; externally peer reviewed.

Open Access This is an Open Access article distributed in accordance with the Creative Commons Attribution Non Commercial (CC BY-NC 3.0) license, which permits others to distribute, remix, adapt, build upon this work non-commercially, and license their derivative works on different terms, provided the original work is properly cited and the use is non-commercial. See: http://creativecommons.org/ licenses/by-nc/3.0/

\section{REFERENCES}

1 World Health Organization. Commission on Social Determinants of Health: final report. 2008. http://www.who.int/social_determinants/thecommission/finalreport/en/ index.html (accessed 26 Jul 2011)

2 Petticrew M, Whitehead M, Macintyre SJ, et al. Evidence for public health policy on inequalities: 1: the reality according to policymakers. J Epidemiol Community Health 2004:58:811-16.

3 Petticrew M. 'More research needed': plugging gaps in the evidence base on health inequalities. Eur J Public Health 2007;17:411-13.

4 Hawe P, Potvin L. What is population health intervention research? Can J Public Health 2009;100(Suppl I):8-14.

5 Edwards N, Di Ruggiero E. Exploring which context matters in the study of health inequities and their mitigation. Scand J Public Health 2011;39:43-9.

6 Wolfson MC. POHEM — a framework for understanding and modelling the health of human populations. World Health Statist Q 1994;47:157-76.

7 Diez Roux AV. Complex systems thinking and current impasses in health disparities research. Am J Public Health 2011;101:1627-34

8 El-Sayed AM, Scarborough P, Seemann L, et al. Social network analysis and agentbased modeling in social epidemiology. Epidemiol Perspect Innovations 2012;9:1.

9 Harper S, Lynch J, Smith GD. Social determinants and the decline of cardiovascular diseases: understanding the links. Ann Rev Public Health 2011:32:39-69.

10 Whitehead M. A typology of actions to tackle social inequalities in health. J Epidemiol Community Health 2007:61:473-8. 
11 Feuer EJ, Etzioni R, Cronin KA, et al. The use of modeling to understand the impact of screening on U.S. mortality: examples from mammography and PSA testing. Stat Methods Med Res 2004;13:421-42.

12 Galea S, Riddle M, Kaplan GA. Causal thinking and complex system approaches in epidemiology. Int J Epidemiol 2010;39:97-106.

13 Ness RB, Koopman JS, Roberts MS. Causal system modeling in chronic disease epidemiology: a proposal. Ann Epidemiol 2007;17:564-8.

14 Hernan MA, Taubman SL. Does obesity shorten life? The importance of well-defined interventions to answer causal questions. Int J Obes (Lond) 2008;32 (Suppl 3):S8-14.

15 Petticrew $M$, Tugwell $P$, Kristjansson $E$, et al. Damned if you do, damned if you don't: subgroup analysis and equity. J Epidemiol Community Health 2012;66:95-8.

16 Harper S, Strumpf EC. Social epidemiology: questionable answers and answerable questions. Epidemiology 2012;23:795-8.

17 Levy DT, Mabry PL, Wang YC, et al. Simulation models of obesity: a review of the literature and implications for research and policy. Obes Rev 2011:12:378-94.

18 Brisson M, Edmunds WJ. Impact of model, methodological, and parameter uncertainty in the economic analysis of vaccination programs. Med Decis Making 2006;26:434-46

19 Kopec JA, Fines P, Manuel DG, et al. Validation of population-based disease simulation models: a review of concepts and methods. BMC Public Health 2010;10:710.

20 Ford ES, Capewell S. Proportion of the decline in cardiovascular mortality disease due to prevention versus treatment: public health versus clinical care. Ann Rev Public Health 2011;32:5-22.

21 Levy DT, Bauer JE, Lee HR. Simulation modeling and tobacco control: creating more robust public health policies. Am J Public Health 2006;96:494-8.

22 Milstein $B$, Homer J, Briss $P$, et al. Why behavioral and environmental interventions are needed to improve health at lower cost. Health Aff (Millwood) 2011:30:823-32.

23 Wilson PW, D'Agostino RB, Levy D, et al. Prediction of coronary heart disease using risk factor categories. Circulation 1998;97:1837-47.

24 Manuel DG, Rosella LC. Commentary: assessing population (baseline) risk is a cornerstone of population health planning-looking forward to address new challenges. Int J Epidemiol 2009;39:380-2.

25 Fiscella K, Tancredi D. Socioeconomic status and coronary heart disease risk prediction. JAMA 2008;300:2666-8.

26 Fiscella K, Tancredi D, Franks P. Adding socioeconomic status to Framingham scoring to reduce disparities in coronary risk assessment. Am Heart J 2009;157:988-94
27 Hippisley-Cox J, Coupland C, Vinogradova Y, et al. Performance of the QRISK cardiovascular risk prediction algorithm in an independent UK sample of patients from general practice: a validation study. Heart 2008;94:34-9.

28 Braveman PA, Cubbin C, Egerter $S$, et al. Socioeconomic status in health research: one size does not fit all. JAMA 2005:294:2879-88.

29 Tugwell $P$, de Savigny $D$, Hawker $G$, et al. Applying clinical epidemiological methods to health equity: the equity effectiveness loop. BMJ 2006;332:358-61.

30 Bambra C, Gibson M, Sowden A, et al. Tackling the wider social determinants of health and health inequalities: evidence from systematic reviews. J Epidemiol Community Health 2010:64:284-91.

31 Tugwell $P$, Petticrew $M$, Kristjansson $E$, et al. Assessing equity in systematic reviews: realising the recommendations of the Commission on Social Determinants of Health. BMJ 2010;341:c4739.

32 Main C, Thomas S, Ogilvie D, et al. Population tobacco control interventions and their effects on social inequalities in smoking: placing an equity lens on existing systematic reviews. BMC Public Health 2008:8:178.

33 Lorenc $T$, Petticrew M, Welch V, et al. What types of interventions generate inequalities? Evidence from systematic reviews. J Epidemio/ Community Health 2013;67:190-3.

34 Bajekal M, Scholes S, Love $\mathrm{H}$, et al. Analysing recent socioeconomic trends in coronary heart disease mortality in England, 2000-2007: a population modelling study. PLoS Med 2012;9:e1001237.

35 Hoffmann R, Eikemo TA, Kulhanova I, et al. The potential impact of a social redistribution of specific risk factors on socioeconomic inequalities in mortality: illustration of a method based on population attributable fractions. J Epidemiol Community Health 2013:67:56-62.

36 Alvarado BE, Harper S, Platt RW, et al. Would achieving healthy people 2010's targets reduce both population levels and social disparities in heart disease? Circ Cardiovasc Qual Outcomes 2009;2:598-606.

37 Benach J, Malmusi D, Yasui Y, et al. A new typology of policies to tackle health inequalities and scenarios of impact based on Rose's population approach. $J$ Epidemiol Community Health 2013;67:286-91.

38 Kivimaki M, Shipley MJ, Ferrie JE, et al. Best-practice interventions to reduce socioeconomic inequalities of coronary heart disease mortality in UK: a prospective occupational cohort study. Lancet 2008:372:1648-54.

39 Bleich SN, Jarlenski MP, Bell CN, et al. Health inequalities: trends, progress, and policy. Ann Rev Public Health 2012:33:7-40.

40 King NB, Harper $S$, Young ME. Use of relative and absolute effect measures in reporting health inequalities: structured review. BMJ 2012;345:e5774. 